\title{
ON A BERGMAN-WHITTAKER TYPE OPERATOR IN FIVE OR MORE VARIABLES ${ }^{1}$
}

\author{
DEAN K. KUKRAL
}

Abstract. It is shown that there cannot exist a simple generating function for homogeneous harmonic polynomials in five or more variables similar to those known to exist for three and four variables. Thus there is no simple immediate generalization of the three dimensional Bergman-Whittaker operator (and Gilbert's four dimensional operator) to five or more dimensions.

The classical Bergman-Whittaker integral operator [5, p. 50] maps analytic functions of two complex variables onto solutions of,

$$
\Delta_{n} u=0
$$

where $n=3$ and $\Delta_{n}$ represents the $n$-dimensional Laplacian. Considerable work has been undertaken to generalize this operator to more general equations (see for example the paper by Colton [1]). To a large degree the success of the techniques involved hinges upon the existence of a certain "auxiliary variable,"

$$
\mu=x_{1}+\zeta\left(x_{2}+i x_{3}\right)+\zeta^{-1}\left(-x_{2}+i x_{3}\right) .
$$

This auxiliary variable has the property $[3$, p. 540] that if one sets

$$
\mu^{N}=\sum_{i=-N}^{N} \zeta^{i} h_{N, i}\left(x_{1}, x_{2}, x_{3}\right), \quad N=1,2, \cdots,
$$

then the set, $\left\{h_{N, i}\right\}$, forms a maximal linearly independent set of $N$ th degree homogeneous harmonic polynomials in the variables $x_{1}, x_{2}$, and $x_{3}$.

For the four dimensional case $(n=4)$ of equation (1) Gilbert [5, p. 75] has devised the auxiliary variable,

(4) $\mu=\left(x_{1}+i x_{2}\right)+\eta^{-1} \zeta^{-1}\left(x_{1}-i x_{2}\right)+\zeta^{-1}\left(x_{3}+i x_{4}\right)+\eta^{-1}\left(-x_{3}+i x_{4}\right)$

Received by the editors July 5, 1972.

AMS (MOS) subject classifications (1970). Primary 35J05, 35J40; Secondary 31B05.

${ }^{1}$ Research sponsored by the Air Force Office at Scientific Research Air Force Systems Command, USAF, under AFOSR Grant Nos. 1206-67 and 71-2205A. The United States Government is authorized to reproduce and distribute reprints for Government purposes notwithstanding any copyright notation hereon.

(c) American Mathematical Society 1973 
which has the property that if one sets

$$
\mu^{N}=\sum_{i=0}^{N} \sum_{j=0}^{N} \eta^{-i \zeta^{-j}} h_{N, i, j}\left(x_{1}, x_{2}, x_{3}, x_{4}\right)
$$

then the $\left\{h_{N, i, j}\right\}$ form a maximal linearly independent set of $N$ th degree homogeneous harmonic polynomials in $x_{1}, x_{2}, x_{3}$, and $x_{4}$. Gilbert's auxiliary variable has been used extensively for developing integral operator solutions of four dimensional elliptic equations (see for example [2] or [6, p. 92]).

It is clear that if such auxiliary variables could be developed for higher dimensions then much of the work already accomplished could be easily extended to higher dimensional elliptic equations. Attempts have been made (see for example [5, p. 82] or [6, p. 164]) to develop such higher dimensional auxiliary variables, but they have not been entirely satisfactory.

We show in this paper that there does not exist such an auxiliary variable for dimensions $n \geqq 5$, so that the techniques such as those employed by Bergman, Gilbert, and Colton cannot be extended to higher dimensions without major modifications.

THEOREM. There is no variable, $\mu$, which, in the sense of BergmanWhittaker (for $n=3$ ) and Gilbert (for $n=4)$, generates the homogeneous linearly independent harmonic polynomials in $n \geqq 5$ variables.

Proof. Suppose on the contrary that there exists

$$
\mu=A_{1} N_{1}+\cdots+A_{n} N_{n}
$$

where the set $\left\{A_{j}\right\}$ is a set of nontrivial linearly independent homogeneous harmonic polynomials of degree one in the variables $x_{1}, \cdots, x_{n}$, and the $\left\{N_{i}\right\}$ are independent products (sums are not allowable if we are to have a $\mu$ "in the sense of Bergman-Whittaker") of $\zeta_{1}, \zeta_{2}, \cdots, \zeta_{p}$ and/or $1 / \zeta_{1}, \cdots$, $1 / \zeta_{p}(n=p+2)$. We must have that $\mu^{m}$ for $m \geqq 0$ is harmonic and in particular $\mu^{2}$ is harmonic,

$$
\mu^{2}=A_{1}^{2} N_{1}^{2}+2 A_{1} A_{2} N_{1} N_{2}+\cdots+A_{n}^{2} N_{n}^{2} .
$$

In equation (7) there can be at most

$$
n+(n-1)+\cdots+3+2+1=n(n+1) / 2
$$

distinct terms. But there are

$$
h(2, p)=h(2, n-2)=(n-1)(n+2) / 2
$$

homogeneous linearly independent harmonic polynomials of degree two 
in $n$ variables [4, V.II, p. 237]. And

$$
n(n+1) / 2-(n-1)(n+2) / 2=1 .
$$

So in order to have the proper number of polynomials of degree two as coefficients of the $N_{i} N_{j}$ in $\mu^{2}$, exactly two terms must combine, no more and no less (i.e. $N_{i} N_{j}=N_{l} N_{m}$ for $i \neq l, m$ and $j \neq l, m$ once and only once).

Since we started with ( $n \geqq 5)$ distinct $A_{j}$, at least one term, which we can without loss of generality take to be $A_{1}$, will be such that the set $\left\{A_{1} A_{j} \mid j=\right.$ $1, \cdots, n\}$ is a set of $n$ homogeneous linearly independent harmonic polynomials of degree two, i.e. each member of the set will appear as a distinct coefficient in $\mu^{2}$. Let $A_{j}=a_{j, 1} x_{1}+a_{j, 2} x_{2}+\cdots+a_{j, n} x_{n}$. Since the members of the set $\left\{A_{j}\right\}$ are nontrivial and linearly independent, the $n$ vectors of the set, $\left\{\left(a_{j, 1}, a_{j, 2}, \cdots, a_{j, n}\right): j=1, \cdots, n\right\}$ are a basis for $C^{n}$. But the condition that $A_{1} A_{j}$ is harmonic for $j=1, \cdots, n$ is equivalent to the condition that the inner products vanish,

$$
\left\langle\left(\bar{a}_{1,1}, \cdots, \bar{a}_{1, n}\right),\left(a_{j, 1}, \cdots, a_{j, n}\right)\right\rangle=0
$$

for $j=1, \cdots, n$. This means that the vector, $\left(\bar{a}_{1,1}, \cdots, \bar{a}_{1, n}\right)$, is orthogonal to every member of a basis for $C^{n}$. It follows that $A_{1} \equiv 0$ which is a contradiction.

\section{REFERENCES}

1. D. Colton, Integral operators for elliptic equations in three independent variables. I, II, Applicable Analysis (to appear).

2. - Integral operators for elliptic equations in four independent variables, SIAM J. Math. Anal. 3 (1972), 401-412.

3. R. Courant and D. Hilbert, Methods of mathematical physics. Vol. I, Interscience, New York, 1953. MR 16, 426.

4. A. Erdélyi et al., Higher transcendental functions. Vol. II, McGraw-Hill, New York, 1953. MR 15, 419.

5. R. P. Gilbert, Function theoretic methods in partial differential equations, Math. in Sci. and Engineering, vol. 54, Academic Press, New York, 1969. MR 39 \#3127.

6. D. K. Kukral, Ph.D. Thesis, Indiana University, Bloomington, Ind., 1972.

Department of Mathematics, Indiana University, Bloomington, Indiana 47401

Current address: Department of Mathematics, Wichita State University, Wichita, Kansas 67206 